\title{
Modification of the masses of the lightest neutral mesons in a hadronic medium under an external magnetic field
}

\author{
R. M. Aguirre \\ Departamento de Fisica, Facultad de Ciencias Exactas, Universidad Nacional de La Plata, \\ and IFLP, UNLP-CONICET, C.C. 67, 1900 La Plata, Argentina \\ (Received 5 September 2017; published 16 November 2017)
}

\begin{abstract}
The effective masses of the neutral mesons in a hadronic medium and under an external magnetic field are evaluated as functions of the baryonic density and the field intensity. For this purpose, the meson polarization is evaluated in the one-loop approximation using a quantum hadrodynamics model which includes $\pi, \sigma, \omega$, and $\rho$ mesons. The propagators of the baryons include the full effect of the coupling to the magnetic field through their charges and their anomalous magnetic moments. Within the range of magnetic intensities considered here $10^{17} \mathrm{G}<B<10^{19} \mathrm{G}$, the dependence on $B$ is moderate for the pion and the longitudinal component of the $\omega$ meson and negligible for the remaining mesons.
\end{abstract}

DOI: 10.1103/PhysRevD.96.096013

\section{INTRODUCTION}

The physics of matter subject to strong magnetic fields presents multiple aspects, which is why it has been widely studied in the past [1,2], and new facets are always being investigated.

Among the different empirical manifestations of very strong magnetic fields, two situations have received particular attention in recent years. On the one hand, the presence of intense magnetic fields has been deduced from the observational data of certain compact stars which have been generally included within the magnetar model $[3,4]$. The sustained x-ray luminosity in the soft $(0.5-10 \mathrm{keV})$ or hard (50-200 keV) spectrum and the bursting activity of these objects have been attributed to the dissipation and decay of very strong fields. The intensity of these fields has been estimated around $10^{15} \mathrm{G}$ at the star surface, but could reach much higher values in the dense interior of the star. The origin of the magnetism, however, is still under debate [5-7].

Furthermore, extreme magnetic intensities are expected in heavy ion collisions [8-10]. Experimental evidence of this fact is the preferential emission of charged particles along the direction of the magnetic field for noncentral heavy ion collisions, due to magnetic intensities $e B \sim$ $10^{2} \mathrm{MeV}^{2}$ [8]. In spite of its evanescent character, the strong field could influence the hadronization process [11].

The effect of external magnetic fields on the quark structure of mesons has intensively been studied in recent years by using lattice QCD [12], QCD sum rules for heavy mesons [13], the nonrelativistic potential for heavy mesons [14], an effective Hamiltonian with QCD basis [15], and the Nambu and Jona-Lasinio model [16,17]. Some of these descriptions do not consider quark confinement explicitly, and others lack the interaction among hadrons.

On the other hand, the use of effective hadronic models to study pions [18-23] as well as vector mesons [24,25] takes account of the hadronic environment although it does not include the substructure dynamics.

It is reasonable that light mesons experience more noticeably the effects of an external magnetic field if $e B \sim$ $m_{\pi}^{2}\left(B \sim 3 \times 10^{18} \mathrm{G}\right)$. Furthermore, as the neutral mesons do not couple directly with the magnetic field, any change in their dynamical properties is due to their interactions with the hadronic medium.

The aim of this work is to investigate the effect of a uniform external field on the properties of the lightest neutral meson. In our approach, the magnetic field is treated as a classical external field; therefore, we neglect electromagnetic quantum corrections. Otherwise, the mixing of neutral pions and photons could be a source of level repulsion.

We consider the magnetic intensity, the baryonic number density, and the isospin composition of matter as relevant parameters.

A successful description of the dense hadronic environment has been given by a covariant model of the hadronic interaction known as quantum hadrodynamics (QHD) [26]. It has been used to study the structure of neutron stars and particularly to analyze hadronic matter in the presence of an external magnetic field [27-35]. In this formulation the elementary degrees of freedom are hadronic fields, regarded as structureless particles. For this reason when the quark dynamics is expected to manifest, additional input should be included in the model. However, QHD is an adequate tool to analyze many-body effects in the hadronic medium for a wide range of applications.

The versatility of QHD allows the inclusion of the intrinsic magnetic moments in a covariant way. Due to the strength of the baryon-meson couplings, the mean field approximation (MFA) is usually employed. Within this approach the meson fields are replaced by their expectation values and assimilated to a quasi-particle picture of the baryons. Finally the meson mean values are obtained by solving the classical meson equations taking as sources the 
baryonic currents. This scheme is conceptually clear and easy to implement.

We use here a model including pions, $\sigma, \omega$ and $\rho$ mesons in order to evaluate the meson polarization in the one loop approximation. The effective mass of the mesons in the hadronic medium is defined and analyzed at zero temperature for a wide range of densities $0<n_{B}<3 n_{0}$, with $n_{0}$ the saturation density of nuclear matter, and magnetic intensities $10^{16} \mathrm{G} \leq B \leq 10^{19} \mathrm{G}$. Two different isospin composition of matter are considered, isospin symmetric nuclear matter and neutral nuclear matter in equilibrium against beta decay.

As mentioned before, the model is not able to describe the consequences of strong magnetic fields on the quark structure. Hence we will not consider effects like the mixing of quark-antiquark bound states induced by an external field at zero baryonic density as reported in recent works [36].

The main contribution to our calculations comes from a ring diagram of the nucleon propagator, which includes the full effect of the coupling of the external field through the electric charge and the anomalous magnetic moment. The significative role played by the intrinsic magnetic moments of the hadrons has been pointed out for the evaluation of bulk properties of dense nuclear matter under strong magnetic fields [30-34].

The propagator of a proton with anomalous magnetic moment immersed in a uniform magnetic field has been presented in [20], and extended to finite density and temperature in [22].

This work is organized as follows. In the next section the one-loop polarization insertion is presented. The results and discussion are given in Sec. III, and the conclusions are shown in Sec. IV.

\section{IN-MEDIUM MESON POLARIZATION INSERTION}

The effective model for the hadronic interaction is given by

$$
\begin{aligned}
\mathcal{L}= & \sum_{a=n, p} \bar{\Psi}^{a}\left[\gamma_{\mu}\left(i \partial^{\mu}-q_{a} A^{\mu}+g_{\omega} \omega^{\mu}+\frac{g_{\rho}}{2} \boldsymbol{\tau} \cdot \boldsymbol{\rho}^{\mu}-\frac{g_{A}}{2 f_{\pi}} \gamma_{5} \boldsymbol{\tau} \cdot \partial^{\mu} \phi-\frac{1}{4 f_{\pi}^{2}} \boldsymbol{\tau} \cdot \boldsymbol{\phi} \times \partial^{\mu} \boldsymbol{\phi}\right)-m_{0}+g_{\sigma} \sigma+\frac{\kappa_{a}}{2} \sigma^{\mu \nu} \mathcal{F}_{\mu \nu}\right] \Psi^{a} \\
& -\frac{A}{3} \sigma^{3}-\frac{B}{4} \sigma^{4}+\frac{C}{4}\left(\omega_{\mu} \omega^{\mu}\right)^{2}+D \boldsymbol{\rho}_{\lambda} \cdot \boldsymbol{\rho}^{\lambda} \omega_{\mu} \omega^{\mu}+\mathcal{L}_{M}
\end{aligned}
$$

here $\mathcal{L}_{M}$ stands for the free mesons part, and only the lowest lying baryons are considered with anomalous magnetic moment represented by $\kappa_{a}$. The interaction includes one and two pion vertices, and the self-interaction of the $\sigma$ and $\omega$ mesons, together with the $\omega-\rho$ coupling $[37,38]$.

In our approach, the fundamental state of matter is given by a mean field approach (MFA), which is equivalent to include the tadpole diagram [see Fig. 1(a)] in a selfconsistent solution but neglecting divergent contributions coming from the Dirac sea. At this step it is assumed that meson propagation is not modified by the hadronic interaction. The effect of the magnetic field, instead, is fully included for both meson and nucleon propagators. It can be verified that pions do not contribute to the tadpole diagram, since the pion-nucleon vertices depend on the transferred pion momentum. Furthermore, the neutral mesons $\pi^{0}, \sigma, \omega_{\mu}$ and $\rho_{\mu}^{0}$ are not affected directly by the magnetic field.

At this step, a quasiparticle picture is obtained for the nucleons, with effective mass $m=m_{0}-g_{\sigma} S$, and energy spectra $p_{0}^{(a)}=g_{\omega} W+g_{\rho} R I_{a} \pm E_{a}$, with

$$
\begin{aligned}
& E_{1}=\sqrt{p_{z}^{2}+\left(\Delta_{n}-s \kappa_{1} B\right)^{2}} \\
& \Delta_{n}=\sqrt{m^{2}+2 n q B}
\end{aligned}
$$$$
I_{1}=1 \text { for protons, and }
$$

$$
\begin{aligned}
E_{2} & =\sqrt{p_{z}^{2}+\left(\Delta-s \kappa_{2} B\right)^{2}} \\
\Delta & =\sqrt{m^{2}+p_{x}^{2}+p_{y}^{2}}
\end{aligned}
$$

$I_{2}=-1$, for neutrons. The index $s= \pm 1$ indicates spin projection along the direction of the uniform magnetic field, and the discrete index $n$ for protons comes from the Landau quantization. It must be remembered that the lowest Landau level $n=0$ admits only $s=1$.

Furthermore, the quantities $S, W$, and $R$ correspond, respectively, to the in-medium expectation values of the $\sigma$ and timelike components of $\omega$ and $\rho^{0}$ mesons [26]. They are related to the hadronic densities by

$$
\begin{aligned}
\left(m_{\sigma}^{2}+A S+B S^{2}\right) S & =g_{\sigma}\left(n_{s 1}+n_{s 2}\right) \\
\left(m_{\omega}^{2}+C W^{2}+2 D R^{2}\right) W & =g_{\omega}\left(n_{1}+n_{2}\right) \\
\left(m_{\rho}^{2}+2 D W^{2}\right) R & =g_{\omega}\left(n_{1}-n_{2}\right),
\end{aligned}
$$

where 


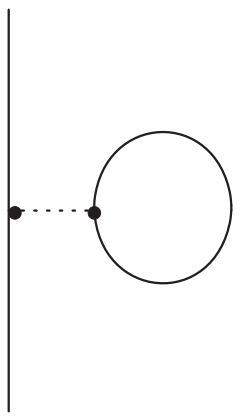

(a)

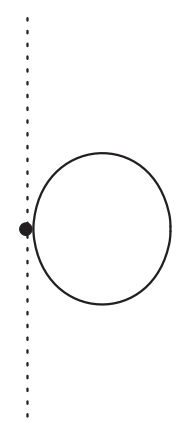

(d)

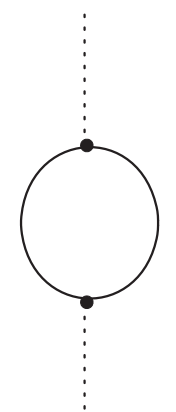

(b)

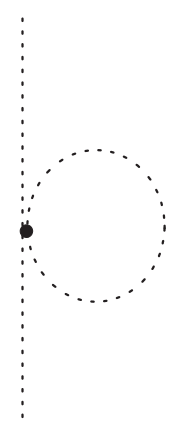

(e)

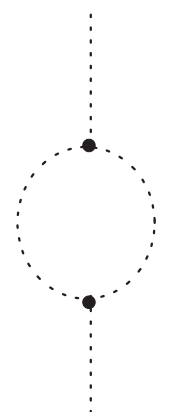

(c)

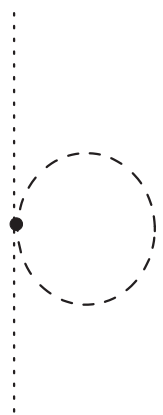

(f)
FIG. 1. The diagrams considered in our Dyson-Schwinger calculations. Solid lines represent fermion propagators, and dashed or dotted lines, the meson ones.

$$
\begin{aligned}
n_{1}= & \frac{q B}{2 \pi^{2}} \sum_{n, s} \int d p_{z}\left[n_{F}\left(E_{1}, \mu_{1}\right)-n_{F}\left(-E_{1}, \mu_{1}\right)\right] \\
n_{2}= & \sum_{s} \int \frac{d^{3} p}{(2 \pi)^{3}}\left[n_{F}\left(E_{2}, \mu_{2}\right)-n_{F}\left(-E_{2} \cdot \mu_{2}\right)\right] \\
n_{s 1}= & \frac{q B}{2 \pi^{2}} m \sum_{n, s} \int d p_{z} \frac{\Delta_{n}+s \kappa_{1} B}{E_{1} \Delta_{n}} \\
& \times\left[n_{F}\left(E_{1}, \mu_{1}\right)+n_{F}\left(-E_{1}, \mu_{1}\right)\right] \\
n_{s 2}= & \sum_{s} \int \frac{d^{3} p}{(2 \pi)^{3}} \frac{\Delta+s \kappa_{2} B}{E_{2} \Delta}\left[n_{F}\left(E_{2}, \mu_{2}\right)+n_{F}\left(-E_{2}, \mu_{2}\right)\right]
\end{aligned}
$$

The two first equations relate the conserved baryon number with the chemical potentials $\mu_{a}$.

The nucleon propagators corresponding to this approach are given in [22]. For the sake of completeness, we show here the neutron propagator,

$$
\begin{aligned}
G^{(2)}\left(x^{\prime}, x\right)= & \sum_{s} \int \frac{d^{4} p}{(2 \pi)^{4}} e^{-i p^{\mu}\left(x_{\mu}^{\prime}-x_{\mu}\right)} \Lambda_{s} \\
& \times\left[\frac{1}{p_{0}^{2}-E_{2}^{2}+i \epsilon}+2 \pi i n_{F}\left(p_{0}\right) \delta\left(p_{0}^{2}-E_{2}^{2}\right)\right],
\end{aligned}
$$

where

$$
\Lambda_{s}=\frac{s}{2 \Delta} i \gamma^{1} \gamma^{2}\left[\not h+i \gamma^{1} \gamma^{2}\left(s \Delta-\kappa_{2} B\right)\right]\left(\not \gamma+m+i s \Delta \gamma^{1} \gamma^{2}\right),
$$

and the proton propagator

$$
\begin{aligned}
G^{(1)}\left(x^{\prime}, x\right)= & e^{i \Phi} \int \frac{d^{4} p}{(2 \pi)^{4}} e^{-i p^{\mu}\left(x_{\mu}^{\prime}-x_{\mu}\right)} \\
& \times\left[G_{0}(p)+\sum_{n, s} G_{n s}(p)\right],
\end{aligned}
$$

where the phase factor $\Phi=q B\left(x+x^{\prime}\right)\left(y^{\prime}-y\right) / 2$ embodies the gauge fixing. We have separated the lowest Landau level contribution,

$$
\begin{aligned}
G_{0}(p)= & 2 e^{-p_{\perp}^{2} / q B} \Lambda_{0} \\
& \times\left[\frac{1}{p_{0}^{2}-E_{0}^{2}+i \epsilon}+2 \pi i n_{F}\left(p_{0}\right) \delta\left(p_{0}^{2}-E_{0}^{2}\right)\right] \\
\Lambda_{0}= & \left(\not h+m-\kappa_{1} B\right) \Pi^{(+)},
\end{aligned}
$$

from the higher Landau levels contributions,

$$
\begin{aligned}
G_{n s}(p)= & e^{-p_{\perp}^{2} / q B} \Lambda_{n s} \\
& \times\left[\frac{1}{p_{0}^{2}-E_{n s}^{2}+i \epsilon}+2 \pi i n_{F}\left(p_{0}\right) \delta\left(p_{0}^{2}-E_{n s}^{2}\right)\right] \\
\Lambda_{n s}= & (-1)^{n} \frac{\Delta_{n}+s m}{\Delta_{n}} \\
& \times\left\{\left(\not h-\kappa_{1} B+s \Delta_{n}\right) \Pi^{(+)} L_{n}\left(2 p_{\perp}^{2} / q B\right)\right. \\
& -\left(\not h+\kappa_{1} B-s \Delta_{n}\right) \Pi^{(-)} \frac{s \Delta_{n}-m}{s \Delta_{n}+m} L_{n-1}\left(2 p_{\perp}^{2} / q B\right) \\
& +\left[\not h+i \gamma_{1} \gamma_{2}\left(s \Delta_{n}-\kappa_{1} B\right)\right] i \gamma^{1} \gamma^{2} \not s \frac{s \Delta_{n}-m}{2 p_{\perp}^{2}} \\
& \left.\times\left[L_{n}\left(2 p_{\perp}^{2} / q B\right)-L_{n-1}\left(2 p_{\perp}^{2} / q B\right)\right]\right\},
\end{aligned}
$$

where $L_{m}$ stands for the Laguerre polynomial of order $m$, and $p_{\perp}^{2}=p_{x}^{2}+p_{y}^{2}$. For this set of equations, the notation $\not h=p_{0} \gamma^{0}-p_{z} \gamma^{3}, \not p=-p_{x} \gamma^{1}-p_{y} \gamma^{2} \Pi^{( \pm)}=\left(1 \pm i \gamma^{1} \gamma^{2}\right) / 2$ is introduced.

The results for these propagators combine the gauge invariance of the proper time method [39] with the momentum representation of [40] and furthermore include the contributions of the anomalous magnetic moments. The extension to finite densities and temperatures [41] has been made in the context of the real time formalism of thermofield dynamics; however, we only exhibit the $(1,1)$ component which suffices for the present calculations at zero temperature.

The magnetic field has a direct coupling to the charged mesons, and there are also quantum corrections which, for the model proposed and at the one loop level are described 
by the diagrams of Figs. 1(b)-1(f). The cases (d)-(f) are first order and come from the two-pion coupling to nucleons (d), the self-interaction of fourth order, either $\sigma$ or $\omega$ (e), and the $\omega-\rho$ coupling (f). The two last cases give zero contribution after regularization. On the other hand, the diagrams (b) and (c) are second order. The third-order $\sigma$ self-interaction gives rise to (c), which is divergent and needs to be regularized. Finally, the nucleon ring diagram (b), as well as the case (d), contain divergent contributions coming from the Dirac sea which will be omitted in our approach.

The finite contribution of diagram (c) is obtained by dimensional regularization and substraction at the point $\mathrm{p}^{2}=m_{\sigma}^{2}$, which is given by

$$
\begin{aligned}
\Pi_{\sigma}(\mathbf{p})= & \left(\frac{A}{12 \pi}\right)^{2}\left[\sqrt{\frac{4 m_{\sigma}^{2}}{\mathrm{p}^{2}}-1} \arctan \left(\frac{4 m_{\sigma}^{2}}{\mathrm{p}^{2}}-1\right)^{-1 / 2}\right. \\
& \left.-\frac{\pi}{2 \sqrt{3}}\right]
\end{aligned}
$$

within the regime $0<\mathrm{p}^{2}<4 m_{\sigma}^{2}$.

The correction (d) to the pion propagation has been evaluated in Ref. [22] and gives zero contribution for the neutral pion.

Finally, we consider the nucleon ring diagram (b). As we are interested in corrections to the neutral mesons, there is no neutron-proton mixing at the vertices. The polarization insertion can be classified as direct,

$$
\begin{aligned}
i \Pi_{\pi}(p)= & \left(\frac{g_{A}}{2 f_{\pi}}\right)^{2} p_{\mu} p_{\nu} \\
& \times \sum_{a=1,2} \int \frac{d^{4} q}{(2 \pi)^{4}} \operatorname{Tr}\left\{\gamma^{\mu} \gamma_{5} G^{(a)}(q) \gamma^{\nu} \gamma_{5} G^{(a)}(q-p)\right\} \\
i \Pi_{\sigma}(p)= & g_{s}^{2} \sum_{a=1,2} \int \frac{d^{4} q}{(2 \pi)^{4}} \operatorname{Tr}\left\{G^{(a)}(q) G^{(a)}(q-p)\right\} \\
i \Pi_{\omega}^{\mu \nu}(p)= & g_{w}^{2} \sum_{a=1,2} \int \frac{d^{4} q}{(2 \pi)^{4}} \operatorname{Tr}\left\{\gamma^{\mu} G^{(a)}(q) \gamma^{\nu} G^{(a)}(q-p)\right\}
\end{aligned}
$$

$i \Pi_{\rho}^{\mu \nu}(p)=\frac{g_{r}^{2}}{4} \sum_{a=1,2} \int \frac{d^{4} q}{(2 \pi)^{4}} \operatorname{Tr}\left\{\gamma^{\mu} G^{(a)}(q) \gamma^{\nu} G^{(a)}(q-p)\right\}$,

which describes the propagation of a given class of mesons, and the mixing components,

$$
\begin{aligned}
i \Pi_{\omega \rho}^{\mu \nu}(p)= & g_{w} \frac{g_{r}}{2} \\
& \times \sum_{a=1,2} I_{a} \int \frac{d^{4} q}{(2 \pi)^{4}} \operatorname{Tr}\left\{\gamma^{\mu} G^{(a)}(q) \gamma^{\nu} G^{(a)}(q-p)\right\}
\end{aligned}
$$

$$
\begin{aligned}
i \Pi_{\omega \rho}(p)= & g_{w} \frac{g_{r}}{2} \\
& \times \sum_{a=1,2} I_{a} \int \frac{d^{4} q}{(2 \pi)^{4}} \operatorname{Tr}\left\{\gamma^{3} G^{(a)}(q) \gamma^{3} G^{(a)}(q-p)\right\}
\end{aligned}
$$

$$
\begin{aligned}
i \Pi_{\pi \omega}(p)= & g_{w} \frac{g_{A}}{2 f_{\pi}} p_{\mu} \\
& \times \sum_{a=1,2} \int \frac{d^{4} q}{(2 \pi)^{4}} \operatorname{Tr}\left\{\gamma^{\mu} \gamma_{5} G^{(a)}(q) \gamma^{3} G^{(a)}(q-p)\right\}
\end{aligned}
$$

$$
\begin{aligned}
i \Pi_{\pi \rho}(p)= & g_{r} \frac{g_{A}}{4 f_{\pi}} p_{\mu} \\
& \times \sum_{a=1,2} I_{a} \int \frac{d^{4} q}{(2 \pi)^{4}} \operatorname{Tr}\left\{\gamma^{\mu} \gamma_{5} G^{(a)}(q) \gamma^{3} G^{(a)}(q-p)\right\},
\end{aligned}
$$

which describes the conversion between different classes of mesons. For the transversal vector mixing of Eq. (6), only $\mu, \nu=1,2$ is possible. Explicit expressions are given in the Appendix.

There are other mixing components not enumerated here, but we focus only on those which are useful for the present calculations. As the meson effective masses will be defined for the dynamical regime $\boldsymbol{p}=0$, we have found that only the mixing components shown above give nonzero contribution.

Thus, there are three blocks of generalized polarization at $\boldsymbol{p}=0$. The sigma component alone on one hand and the transversal $\omega$ and $\rho$ components together with the transversal mix of Eq. (6) on the other hand and, finally, the pion, with the $(3,3)$ component of the $\omega$ and $\rho$ together with the vector longitudinal mixing of Eq. (7) and the pionvector mixing of Eqs. (8) and (9).

For each of these blocks, we define a dielectric function,

$$
\begin{gathered}
\varepsilon_{S}(p)=\mathrm{p}^{2}-m_{\sigma}^{2}-\Pi_{\sigma}(p) \\
\varepsilon_{T}(p)=\operatorname{det} M_{T} \\
\varepsilon_{L}(p)=\operatorname{det} M_{L},
\end{gathered}
$$

where $\Pi_{\sigma}$ consists of the sum of Eqs. (2) and (3) and 


$$
\begin{aligned}
& M_{T}=\left(\begin{array}{cccc}
\mathrm{p}^{2}-m_{\omega}^{2}-\Pi_{\omega}^{11} & -\Pi_{\omega}^{12} & -\Pi_{\omega \rho}^{11} & -\Pi_{\omega \rho}^{12} \\
-\Pi_{\omega}^{21} & \mathrm{p}^{2}-m_{\omega}^{2}-\Pi_{\omega}^{22} & -\Pi_{\omega \rho}^{21} & -\Pi_{\omega \rho}^{22} \\
-\Pi_{\omega \rho}^{11} & -\Pi_{\omega \rho}^{21} & \mathrm{p}^{2}-m_{\rho}^{2}-\Pi_{\rho}^{11} & -\Pi_{\rho}^{12} \\
-\Pi_{\omega \rho}^{21} & -\Pi_{\omega \rho}^{22} & -\Pi_{\rho}^{21} & \mathrm{p}^{2}-m_{\rho}^{2}-\Pi_{\rho}^{22}
\end{array}\right), \\
& M_{L}=\left(\begin{array}{ccc}
\mathrm{p}^{2}-m_{\pi}^{2}-\Pi_{\pi} & -\Pi_{\pi \omega} & -\Pi_{\pi \rho} \\
-\Pi_{\pi \omega} & \mathrm{p}^{2}-m_{\omega}^{2}-\Pi_{\omega}^{33} & -\Pi_{\omega \rho} \\
-\Pi_{\pi \rho} & -\Pi_{\omega \rho} & \mathrm{p}^{2}-m_{\rho}^{2}-\Pi_{\rho}^{33}
\end{array}\right)
\end{aligned}
$$

For given values of the baryonic number density and the field intensity, the equations $\operatorname{Re}\left(\varepsilon_{S, T, L}\right)=0$ evaluated at $\boldsymbol{p}=0$ are equations in $p_{0}$, whose solutions are identified as the effective masses of the mesons. For each equation, we have found multiple solutions-almost all the branches can be traced back at zero density and identified with a definite meson $\pi, \sigma, \omega$ or $\rho$.

In the next section, we show the results obtained for two different configurations of matter-symmetric nuclear matter $\left(n_{1}=n_{2}\right)$ and stellar matter composed of a neutral combination of electrons, protons and neutrons in equilibrium against beta decay.

\section{RESULTS AND DISCUSSION}

In this section, we analyze the effective meson masses for different situations of physical interest. We consider magnetic intensities $10^{16}-10^{19} \mathrm{G}$, a range that can be found in magnetars, and matter at zero temperature and baryonic densities below $0.45 \mathrm{fm}^{-3}$. Under these conditions, strange baryons do not have a significative role; therefore, we only consider protons and neutrons.

For the model parameters, we use the set Z271v6 of Ref. [37], $m_{\sigma}=465 \mathrm{MeV}, m_{\omega}=783 \mathrm{MeV}, m_{\rho}=763 \mathrm{MeV}$, $g_{\sigma}=7.0313, \quad g_{\omega}=8.406, \quad g_{\rho}=10.016, \quad C_{v}=49.941$, $D=283.569, B=63.691$, and $A=1072.37 \mathrm{MeV}$. This parametrization guarantees the binding properties of nuclear matter in the MFA $\left(n_{0}=0.1484 \mathrm{fm}^{-3}, E_{B}=\right.$ $-16.24 \mathrm{MeV}, K=271 \mathrm{MeV}$ ) and the viability of the direct URCA cooling in neutron stars with mass $1.4 M_{\odot}$.

We have added the pion-nucleon vertices, which do not modify the MFA results.

As a first step, we evaluate the MFA at zero temperature, in which case the Fermi occupation number becomes a step function. As a consequence, the Landau levels of the proton are occupied until a well-defined maximum value. At the end of this calculation, we obtain the chemical potentials, the effective nucleon mass, and the maximum Landau level occupied as functions of the magnetic intensity and the baryonic density. The results of the MFA are inserted in the neutron and proton propagators, for evaluating the polarization insertions and the dielectric functions (10)-(12). We first investigate solutions corresponding to $p_{0}<1 \mathrm{GeV}$.
In Fig. 2, the solutions as a function of the baryonic number density for symmetric nuclear matter are shown for several magnetic intensities. The neutral pion branch (a) increases with the density, growing at most $10 \%-$ $15 \%$ for $n / n_{0}=3$. In Ref. [22], the author presented a similar calculation, but neglected the effect of heavier neutral mesons. A comparison of Fig. 6(c) of that reference shows that, for the same conditions, a more pronounced increase of about $15 \%-40 \%$ is obtained. Thus, our first conclusion is that the mix with the longitudinal vector meson channels in the present calculations is responsible for a strong moderation of the rate of growth with density at constant magnetic intensity.

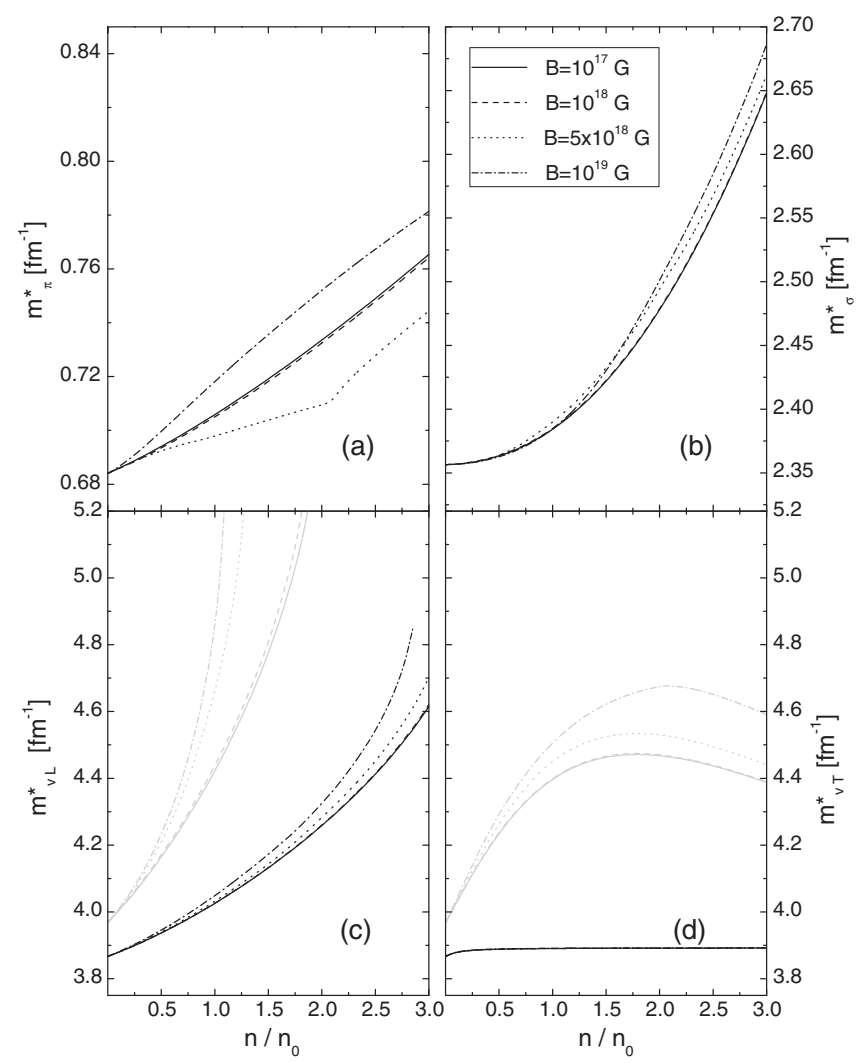

FIG. 2. The effective meson masses as functions of the baryonic number density for several magnetic intensities corresponding to isospin symmetric nuclear matter. 
Returning to Fig. 2(a), it can be observed that the curves for intensities $10^{16}-10^{18} \mathrm{G}$ are very similar. For a fixed density, the slope decreases with $B$ until $B>5 \times 10^{18} \mathrm{G}$, where it increases rapidly. In fact, the highest variation occurs between the curves corresponding to $5 \times 10^{18} \mathrm{G}$ and $10^{19} \mathrm{G}$.

The $\sigma$ meson mass increases with density also, and a mean growth of $15 \%$ is registered at $n / n_{0}=3$. But in contrast with the previous case, these results scarcely depend on the field intensity, showing at most $2 \%$ of dispersion with $B$ at the highest density examined here.

The longitudinal component of the vector mesons also exhibits a monotonous increasing trend with $n$. The growth of the $\rho$ meson mass reaches $20 \%$, while for the $\omega$ meson it exceeds $30 \%$. As can be clearly seen in Fig. 2(c), the dispersion due to the magnetic field increases with the density, reaching $12 \%$ at $n / n_{0} \simeq 1$ for the $\omega$ and $7 \%$ for the $\rho$ at $n / n_{0} \simeq 2.8$. It is interesting that the curves corresponding to $B=10^{19} \mathrm{G}$ exhibit an almost vertical slope for certain densities and the disappearance of the $\rho$ branch after this threshold.

A contrasting result is obtained for the transversal component of the vector mesons [Fig. 2(d)]. The $\rho$ branch is almost constant for the entire range of densities, and the dependence on $B$ is negligible. The transversal $\omega$ branch exhibits at subsaturation densities $\left(n / n_{0}<0.5\right)$ a behavior similar to the corresponding longitudinal component. However, all the curves change their concavity and become decreasing for high enough densities.

In Fig. 3, the same quantities as in Fig. 2 are analyzed, but now for a configuration of matter that can be found in a neutron star, that is, neutrons, protons, and electrons in a homogeneous and neutral compound in equilibrium against beta decay.

In the case of the pion mass [Fig. 3(a)], the main differences with respect to the previous case are that (i) the slope of the curves increases with $B$ for $n / n_{0}>0.75$, and (ii) in the present case, there is a stronger growth for $B>5 \times 10^{18} \mathrm{G}$, which causes an enhancement of $20 \%$ at $n / n_{0}=3$ and $B=10^{19} \mathrm{G}$.

The $\sigma$ branch [Fig. 3(b)] does not exhibit noticeable differences, with the exception of the slight inversion in the ordering of the curves for the $B$ constant.

The longitudinal component of vector mesons [Fig. 3(c)] presents quantitative differences mainly in the $\rho$ branch. A weaker growth with density, but a wider dispersion with $B$ at fixed density, correspond to the present case. The $\omega$ branch keeps a strong growth, exceeding the $1 \mathrm{GeV}$ limit adopted for our analysis at densities around $n / n_{0}=1.5$.

Finally, for the transversal component of the vector mesons [Fig. 3(d)], we can see the $\rho$ branch remains practically invariable, while the $\omega$ branch shows a slightly increased rate of growth with density for $n / n_{0}<1.5$ but finally becomes decreasing for higher densities.

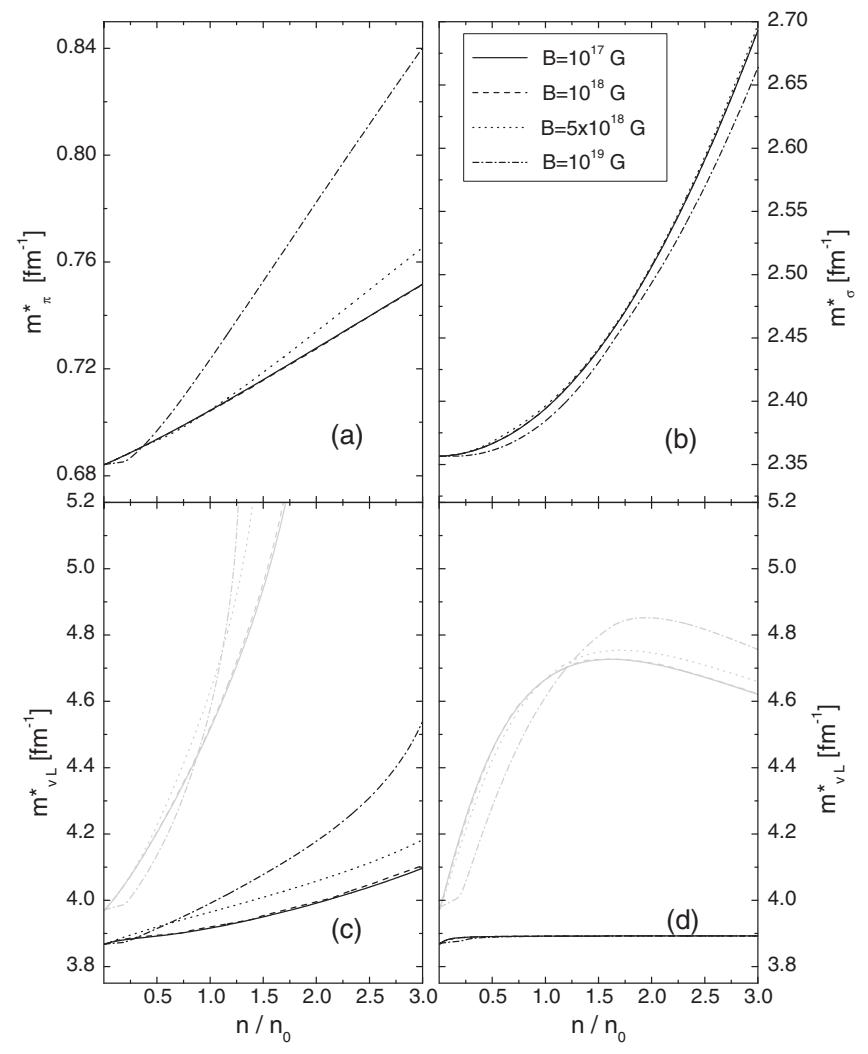

FIG. 3. The effective meson masses as functions of the baryonic number density for several magnetic intensities corresponding to neutral stellar matter.

In Figs. 4 and 5, we show the dependence of the meson masses on the magnetic intensity for isospin symmetric matter at a fixed density $n / n_{0}=1$. In Fig. 4 , we include the results for the intensities $B$ discussed up to this point, but in Fig. 5 we extend our analysis to larger values.

In Fig. 4, we see that only the pion and the longitudinal $\omega$ component have a noticeable variation of roughly $3 \%$, while the others remain almost invariable. Interestingly, the pion mass decreases for low intensities, until it reaches a stationary point at $B \simeq 5 \times 10^{18} \mathrm{G}$ and becomes increasing for stronger fields.

Up to this point, we have examined the results valid for physical interpretation. With the sole purpose of exploring the limits of the approach used, in the following we expand this panorama. In Fig. 5, the range for the masses of resonances increases up to $1.6 \mathrm{GeV}$, and extreme magnetic intensities such that $e B \sim\left(1.5 \Lambda_{\mathrm{QCD}}\right)^{2}$ are considered. Under such conditions, the lowest-lying state is two-folded. It has a low $B$ manifestation, which we identify as the normal pion because it can be continuously traced back at zero density to the empirical value $m_{\pi}$ of the pion mass. It has just been described in Fig. 4, but here is evident that it ends abruptly at $e B \simeq 0.08 \mathrm{GeV}^{2}$. The second branch is identified as the abnormal pion, although it is strictly a resonance of the hadronic system. It starts near $e B \simeq$ $0.05 \mathrm{GeV}^{2}$ with an initial value $25 \%$ below the vacuum 


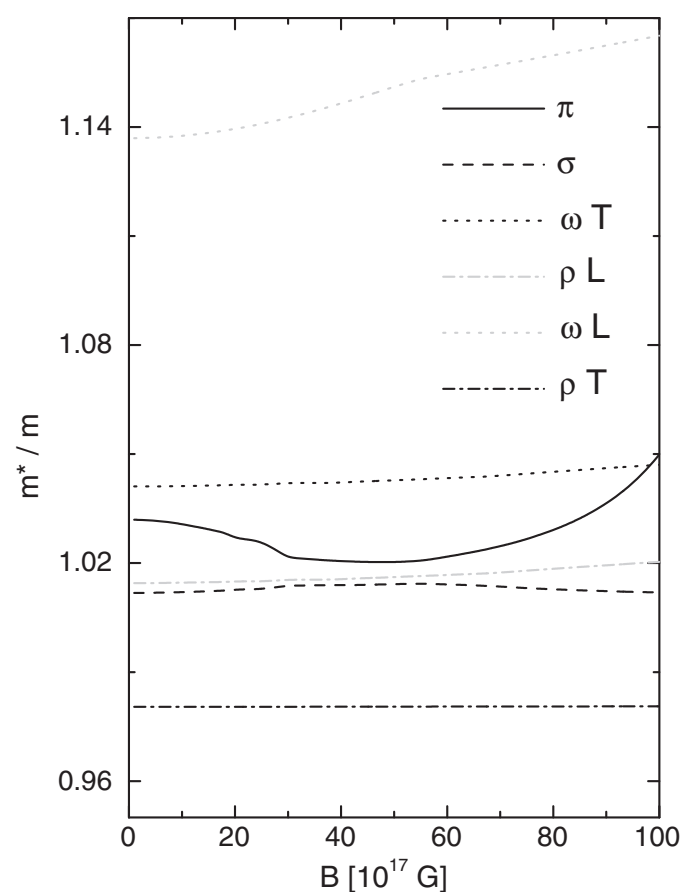

FIG. 4. The effective meson masses as functions of the magnetic intensity for isospin symmetric nuclear matter at the fixed baryonic number density $n=n_{0}$.

value of the pion mass. Both branches coexist while they increase rapidly. For greater intensities, the abnormal branch stabilizes and becomes the only pionic manifestation, with an effective mass slightly below $m_{\pi}$.

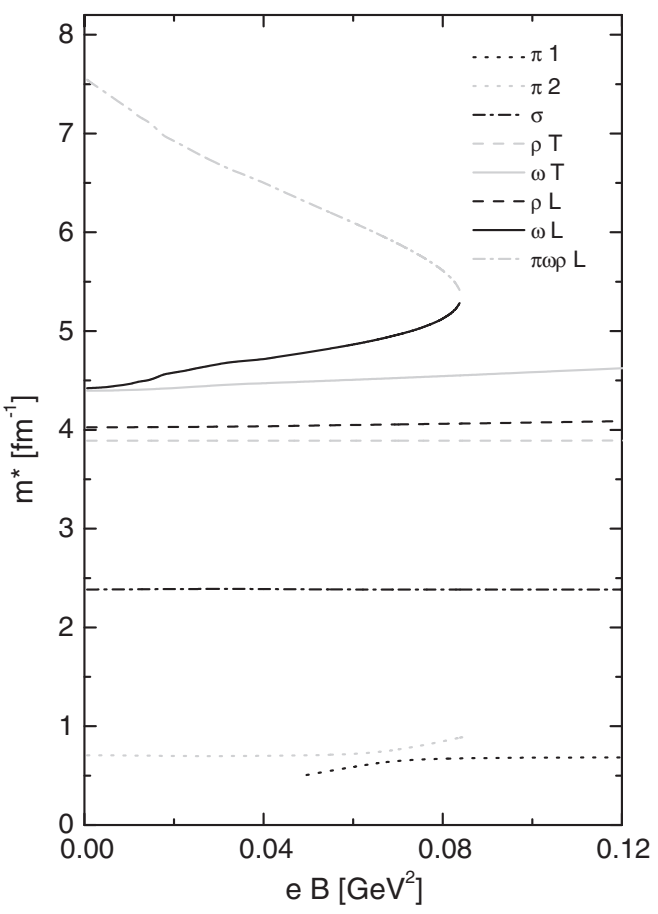

FIG. 5. The same as in Fig. 4, but for a wider magnetic range and an extended scale for the meson masses.
The behavior of the neutral pion mass for a hadronic model with pseudovector coupling in the presence of a magnetic field was discussed in Ref. [21]. Their results correspond to zero baryonic density, so the comparison must be done carefully. In fact, it can be seen in Fig. 2(a), that magnetic effects are weaker as the density approaches to zero. The results of [21] correspond to a monotonous and slight increase with $B$, showing a growth below $1 \%$ at $e B=0.02 \mathrm{GeV}^{2}$. A similar trend can be deduced from Fig. 5(b) of Ref. [22] whose results differ from the present calculations in the inclusion of additional meson fields. Therefore, we conclude that the meson mixing changes considerably the dynamics of the neutral pion field within the range $B<10^{19} \mathrm{G}$ and moderate baryonic densities.

Both components of the neutral $\rho$ meson as well as the $\sigma$ meson masses are really insensitive to the external field showing a smooth, almost constant behavior. The transversal $\omega$ branch is also linearly dependent on the field intensity with a weak slope.

The longitudinal branch of the omega meson collapses near $e B=0.08 \mathrm{GeV}^{2}$. Peculiarly, at this point, it meets the branch corresponding to a heavier partner resonance. The pair displays a specular behavior, the meson mass increases with $B$ while the mass of the partner has the opposite trend. Finally, they coalesce and disappear for a typical intensity.

The present work analyzes the effects on the effective meson masses due to a combination of the external field and the response of the dense hadronic medium. The contributions at zero density of the fermion loops have not been considered. However, different studies have focused on this subject, with different conclusions. At zero density, the neutral pion mass decreases with $B$ within the NJL model [17]. In contrast, the hadronic model of Ref. [21] predicts, under the same conditions, a slight increase. The same NJL treatment predicts an increase of the $\sigma$ meson mass, whose magnitude is considerably greater than for the pion.

\section{CONCLUSIONS}

In this work, an analysis of the effective mass of the lightest neutral mesons in the presence of an external magnetic field has been carried out. The magnitude of the field as well as the density of the hadronic medium have been taken as significative variables, in a zero-temperature treatment. Two different configurations of the hadronic environment have been considered-isospin symmetric nuclear matter and stellar matter. We have focused on strong magnetic fields $10^{16} \mathrm{G} \leq B \leq 10^{19} \mathrm{G}$ and a range of baryonic densities where hyperons are not expected to play a significative role.

The calculations have been made within a covariant model of meson-baryon couplings plus meson self-interactions. The meson polarization has been evaluated in a one-loop self-consistent calculation, but neglecting the divergent contributions from the Dirac sea. The corrections 
due to the baryons have been introduced by using a covariant propagator which includes the full effect of the coupling to the external field through the electric charge and the anomalous magnetic moments.

The analysis of the density dependence shows that the composition of the environment does not modify qualitatively the behavior of the meson masses, and only minor features distinguish between isospin symmetric nuclear matter and stellar matter.

The masses of all the mesons increase monotonically with the density, with the exceptions of the transversal components of the $\omega$ which become decreasing for high densities, and the $\rho$ one, which is almost constant. The increase experienced at the highest density is moderate (around $10 \%$ ) for the $\pi, \sigma$ and transversal $\omega$, considerable for the longitudinal $\rho$ (around 20\%), and important for the longitudinal $\omega$, which exceeds the $30 \%$ in the midrange of densities.

The study of the dependence on the magnetic intensity for isospin symmetric nuclear matter at the characteristic density $n=n_{0}$ in the domain $10^{17} \mathrm{G} \leq B \leq 10^{19} \mathrm{G}$, and effective meson masses below $1 \mathrm{GeV}$, shows a constant mass for the transversal $\rho$, a sustained but scarce increase for the transversal $\omega$, and the longitudinal $\rho$ branches. The $\sigma$ and $\pi$ masses exhibit a nonmonotonous and mirrorlike trend. For low intensities, the $\pi$ mass decreases and the $\sigma$ one increases with $B$; for medium to large intensities, the roles are changed. The largest variance, of roughly $2 \%$, corresponds to the $\pi$ and longitudinal $\omega$.

Further developments for this line of investigation will include the study of thermal effects and of the influence of the internal structure of hadrons in the zero-density state.

\section{ACKNOWLEDGMENTS}

This work has been partially supported by a grant from the Consejo Nacional de Investigaciones Cientificas y Tecnicas, Argentina.

\section{APPENDIX: ONE-LOOP MESON POLARIZATION INSERTIONS}

Here, the formulas for the nucleon loops contributing to the different meson channels are shown, evaluated at zero spatial component of the external momentum $\boldsymbol{p}=0$. For each channel, there is a sum of two contributions, due to neutron and proton loops:

(a) Neutron contributions:

$$
\begin{aligned}
& \operatorname{Re} \Pi_{\pi}\left(p_{0}\right)=\left(\frac{g_{A}}{4 \pi f_{\pi}}\right)^{2} \sum_{s} \int_{0}^{\infty} d t \Theta\left(\tilde{\mu}_{n}-\left|\mathcal{M}_{s}\right|\right)\left(s s^{\prime}+\frac{m^{2}-t}{\Delta^{2}}\right)\left\{\left(\mathcal{M}_{s^{\prime}}^{2}-\mathcal{M}_{s}^{2}\right) \log \left|\frac{\tilde{\mu}_{p}+p_{F n s}}{\tilde{\mu}_{p}-p_{F n s}}\right|\right. \\
& \left.+\frac{p_{0}^{2}-\left(\mathcal{M}_{s}-\mathcal{M}_{s^{\prime}}\right)^{2}}{p_{0} \lambda}\left(\mathcal{M}_{s}+\mathcal{M}_{s^{\prime}}\right)^{2} F(\alpha)\right\} \\
& \operatorname{Re} \Pi_{\sigma}\left(p_{0}\right)=\left(\frac{g_{\sigma}}{4 \pi}\right)^{2} \sum_{s, s^{\prime}} \int_{0}^{\infty} d t \Theta\left(\tilde{\mu}_{n}-\left|\mathcal{M}_{s}\right|\right)\left(1+s s^{\prime} \frac{m^{2}-t}{\Delta^{2}}\right)\left\{\log \left|\frac{\tilde{\mu}_{n}+p_{F s}}{\tilde{\mu}_{n}-p_{F s}}\right|+\frac{p_{0}^{2}-\left(\mathcal{M}_{s}+\mathcal{M}_{s^{\prime}}\right)^{2}}{p_{0} \lambda} F(\alpha)\right\} \\
& \operatorname{Re} \Pi_{\omega}^{11}\left(p_{0}\right)=\left(\frac{g_{\omega}}{4 \pi}\right)^{2} \sum_{s, s^{\prime}} \int_{0}^{\infty} d t \Theta\left(\tilde{\mu}_{n}-\left|\mathcal{M}_{s}\right|\right)\left(1-s^{\prime} s \frac{m^{2}}{\Delta^{2}}\right)\left\{\log \left|\frac{\tilde{\mu}_{n}+p_{F s}}{\tilde{\mu}_{n}-p_{F s}}\right|+s^{\prime} s \frac{p_{0}^{2}-\left(\mathcal{M}_{s}+\mathcal{M}_{s^{\prime}}\right)^{2}}{p_{0} \lambda} F(\alpha)\right\} \\
& \operatorname{Re} \Pi_{\omega}^{12}\left(p_{0}\right)=-\frac{g_{\omega}}{8 \pi} m \sum_{s} s \int_{0}^{\infty} \frac{d t}{\Delta} \frac{\left(2 \kappa_{n} B\right)^{2}-p_{0}^{2}}{p_{0} \lambda}\left\{\Theta\left(-p_{0}^{2}-2 p_{0}\left|s \Delta+\kappa_{n} B\right|-4 s \Delta \kappa_{n} B\right) \Theta\left(\tilde{\mu}_{n}+\frac{p_{0}^{2}+4 s \Delta \kappa_{n} B}{2 p_{0}}\right)\right. \\
& \left.+\Theta\left(\tilde{\mu}_{n}-\frac{p_{0}^{2}-4 s \Delta \kappa_{n} B}{2 p_{0}}\right)\left[1-2 \Theta\left(\tilde{\mu}_{n}+\frac{p_{0}^{2}+4 s \Delta \kappa_{n} B}{2 p_{0}}\right)\right] \Theta\left(p_{0}^{2}-2 p_{0}\left|s \Delta-\kappa_{n} B\right|-4 s \Delta \kappa_{n} B\right)\right\} \\
& \operatorname{Re} \Pi_{\omega}^{33}\left(p_{0}\right)=\left(\frac{g_{\omega}}{\pi}\right)^{2} \sum_{s, s^{\prime}} \int_{0}^{\infty} \frac{d t}{2 p_{0}} \delta_{s s^{\prime}} \Theta\left(\tilde{\mu}_{n}-\left|\mathcal{M}_{s}\right|\right) \frac{\mathcal{M}_{s}^{2}}{\lambda} F(\alpha) \\
& \operatorname{Re} \Pi_{\pi \omega}\left(p_{0}\right)=-\frac{g_{\omega}}{\pi^{2}} \frac{g_{A}}{f_{\pi}} m \sum_{s s^{\prime}} s \delta_{s s^{\prime}} \int_{0}^{\infty} \frac{d t}{2 \Delta} \Theta\left(\tilde{\mu}_{n}-\left|\mathcal{M}_{s}\right|\right) \frac{\mathcal{M}_{s}^{2}}{\lambda} F(\alpha) \\
& F(\alpha)=2 \Theta\left(4 \mathcal{M}_{s}^{2}-\left(p_{0}-\alpha\right)^{2}\right) \arctan \left(\frac{\left(p_{0}-\alpha\right) p_{F s}}{\tilde{\mu}_{n} \lambda}\right)+\Theta\left(\left(p_{0}-\alpha\right)^{2}-4 \mathcal{M}_{s}^{2}\right) \log \left|\frac{\tilde{\mu}_{n} \lambda-\left(p_{0}-\alpha\right) p_{F s}}{\tilde{\mu}_{n} \lambda+\left(p_{0}-\alpha\right) p_{F s}}\right|
\end{aligned}
$$

where $\Delta=\sqrt{m^{2}+t}, \quad \mathcal{M}_{s}=s \Delta-\kappa_{n} B, \quad p_{F s}=\sqrt{\tilde{\mu}_{n}^{2}-\mathcal{M}_{s}^{2}}, \quad \alpha=\left(\mathcal{M}_{s^{\prime}}^{2}-\mathcal{M}_{s}^{2}\right) / p_{0}, \quad$ and $\lambda=\sqrt{\left|4 \mathcal{M}_{s}^{2}-\left(p_{0}-\alpha\right)^{2}\right|}$. Although the domain of integration is not bounded, the relation

$$
\Theta\left(\tilde{\mu}_{n}-\left|\mathcal{M}_{s}\right|\right) \equiv \Theta\left(\left(\tilde{\mu}_{n}+s \kappa_{n} B\right)^{2}-m^{2}-t\right) \Theta\left(\tilde{\mu}_{n}+s \kappa_{n} B-m\right)
$$

valid for the conditions under consideration, establishes an upper limit of integration. 
(b) Proton contributions

$$
\begin{aligned}
& \operatorname{Re}_{\sigma}\left(p_{0}\right)=\left(\frac{g_{\sigma}}{4 \pi}\right)^{2} q B \sum_{s, s^{\prime} ; l, n=0} \delta_{n l} \Theta\left(\tilde{\mu}_{p}-\left|\mathcal{M}_{n s}\right|\right)\left(1+2 \frac{m-s^{\prime} \Delta_{n}}{m+s \Delta_{n}}+\frac{m-s \Delta_{n}}{m+s \Delta_{n}} \frac{m-s^{\prime} \Delta_{n}}{m+s^{\prime} \Delta_{n}}\right) \\
& \times \frac{\Delta_{n}+s m}{\Delta_{n}} \frac{\Delta_{n}+s^{\prime} m}{\Delta_{n}}\left\{\log \left|\frac{\tilde{\mu}_{n}+p_{F s}}{\tilde{\mu}_{n}-p_{F s}}\right|+\frac{p_{0}^{2}-\left(\mathcal{M}_{n s}+\mathcal{M}_{n s^{\prime}}\right)^{2}}{\eta p_{0} \lambda_{l s^{\prime}, n s}} G_{l s^{\prime}, n s}\right\} \\
& \operatorname{Re}_{\pi}\left(p_{0}\right)=\left(\frac{g_{A}}{4 \pi f_{\pi}}\right)^{2} q B \sum_{s, s^{\prime}: l, n=0} \delta_{n l} \Theta\left(\tilde{\mu}_{p}-\left|\mathcal{M}_{n s}\right|\right)\left(1-2 \frac{m-s^{\prime} \Delta_{n}}{m+s \Delta_{n}}+\frac{m-s \Delta_{n}}{m+s \Delta_{n}} \frac{m-s^{\prime} \Delta_{n}}{m+s^{\prime} \Delta_{n}}\right) \\
& \times \frac{\Delta_{n}+s m}{\Delta_{n}} \frac{\Delta_{n}+s^{\prime} m}{\Delta_{n}}\left\{\left(\mathcal{M}_{n s}^{2}-\mathcal{M}_{n s^{\prime}}^{2}\right) \log \left(\frac{\tilde{\mu}_{p}+p_{F n s}}{\tilde{\mu}_{p}-p_{F n s}}\right)+\frac{p_{0}-\alpha_{l s^{\prime}, n s}}{\lambda_{l s^{\prime}, n s}}\left(\mathcal{M}_{n s}+\mathcal{M}_{n s^{\prime}}\right)^{2} G_{l s^{\prime}, n s}\right\} \\
& \operatorname{Re} \Pi_{\omega}^{11}\left(p_{0}\right)=\left(\frac{g_{\omega}}{4 \pi}\right)^{2} q B \sum_{s, s^{\prime} ; l, n=1}\left(\delta_{n+1, l} \frac{\Delta_{n}+s m}{\Delta_{n}} \frac{\Delta_{l}-s^{\prime} m}{\Delta_{l}}+\delta_{n, l+1} \frac{\Delta_{n}-s m}{\Delta_{n}} \frac{\Delta_{l}+s m}{\Delta_{l}}\right) \\
& \times \Theta\left(\tilde{\mu}_{p}-\left|\mathcal{M}_{n s}\right|\right)\left[\log \left|\frac{\tilde{\mu}_{p}+p_{F n s}}{\tilde{\mu}_{p}-p_{F n s}}\right|+\frac{p_{0}^{2}-\left(\mathcal{M}_{n s}+\mathcal{M}_{l s^{\prime}}\right)^{2}}{p_{0} \lambda_{l s^{\prime}, n s}} G_{n s, l s^{\prime}}\right] \\
& \operatorname{Re} \Pi_{\omega}^{12}\left(p_{0}\right)=-\frac{g_{\omega}^{2}}{16 \pi} q B \sum_{s, s^{\prime}, l, n=1}\left(\delta_{n+1, l} \frac{\Delta_{n}+s m}{\Delta_{n}} \frac{\Delta_{l}-s^{\prime} m}{\Delta_{l}}-\delta_{n, l+1} \frac{\Delta_{n}-s m}{\Delta_{n}} \frac{\Delta_{l}+s^{\prime} m}{\Delta_{l}}\right) \\
& \times\left\{\Theta\left(\tilde{\mu}_{p}+\frac{p_{0}-\alpha_{n s, l s^{\prime}}}{2}\right) \Theta\left(-\frac{p_{0}-\alpha_{n s, l s^{\prime}}}{2}-\mathcal{M}_{l s^{\prime}}\right)+\Theta\left(\tilde{\mu}_{p}-\frac{p_{0}+\alpha_{n s, l s^{\prime}}}{2}\right)\right. \\
& \left.\times \Theta\left(\frac{p_{0}+\alpha_{n s, l s^{\prime}}}{2}-\mathcal{M}_{n s}\right)\left[1-2 \Theta\left(\tilde{\mu}_{p}+\frac{p_{0}-\alpha_{n s, l s^{\prime}}}{2}\right)\right]\right\} \frac{p_{0}^{2}-\left(\mathcal{M}_{n s}+\mathcal{M}_{l s^{\prime}}\right)^{2}}{p_{0} \lambda_{l s^{\prime}, n s}} \\
& \operatorname{Re} \Pi_{\omega}^{33}\left(p_{0}\right)=\left(\frac{g_{\omega}}{\pi}\right)^{2} \frac{q B}{p_{0}} \sum_{s, s^{\prime} ; l, n=0} \delta_{n l} \delta_{s s^{\prime}} \Theta\left(\tilde{\mu}_{p}-\left|\mathcal{M}_{n s}\right|\right) \frac{\mathcal{M}_{n s}^{2}}{\lambda_{l s^{\prime}, n s}} G_{l s^{\prime}, n s} \\
& \operatorname{Re} \Pi_{\pi \omega}\left(p_{0}\right)=\frac{g_{\omega}}{\pi^{2}} \frac{g_{A}}{f_{\pi}} q B m \sum_{s s^{\prime}, n=0} s \delta_{s s^{\prime}} \delta_{n l} \Theta\left(\tilde{\mu}_{p}-\left|\mathcal{M}_{n s}\right|\right) \frac{\mathcal{M}_{n s}^{2}}{\Delta_{n} \lambda_{l s^{\prime}, n s}} G_{l s^{\prime}, n s} \\
& G_{l s^{\prime}, n s}=2 \Theta\left(4 \mathcal{M}_{l s^{\prime}}^{2}-\left(p_{0}+\alpha_{l s^{\prime}, n s}\right)^{2}\right) \arctan \left(p_{F l s^{\prime}} \frac{p_{0}+\alpha_{l s^{\prime}, n s}}{\tilde{\mu}_{p} \lambda_{l s^{\prime}, n s}}\right) \\
& +\Theta\left(\left(p_{0}+\alpha_{l s^{\prime}, n s}\right)^{2}-4 \mathcal{M}_{l s^{\prime}}^{2}\right) \log \left|\frac{\tilde{\mu}_{p} \lambda_{l s^{\prime}, n s}-\left(p_{0}+\alpha_{l s^{\prime}, n s}\right) p_{F l s^{\prime}}}{\tilde{\mu}_{p} \lambda_{l s^{\prime}, n s}+\left(p_{0}+\alpha_{l s^{\prime}, n s}\right) p_{F l s^{\prime}}}\right|
\end{aligned}
$$

with $\quad \Delta_{n}=\sqrt{m^{2}+2 n q B}, \quad \mathcal{M}_{n s}=s \Delta_{n}-\kappa_{p} B, \quad p_{F n s}=\sqrt{\tilde{\mu}_{p}^{2}-\mathcal{M}_{n s}^{2}}, \quad \alpha_{l s^{\prime}, n s}=\left(\mathcal{M}_{l s^{\prime}}^{2}-\mathcal{M}_{n s}^{2}\right) / p_{0}, \quad$ and $\quad \lambda_{l s^{\prime}, n s}=$ $\sqrt{\left|4 \mathcal{M}_{l s^{\prime}}^{2}-\left(p_{0}+\alpha_{l s^{\prime}, n s}\right)^{2}\right|}$. In the summations, it must be understood that, for $n=0$, only the case $s=s^{\prime}=1$ must be included.

Furthermore, the following relations are valid for both protons and neutrons,

$$
\begin{aligned}
& \operatorname{Re} \Pi_{\omega}^{22}\left(p_{0}\right)=\operatorname{Re} \Pi_{\omega}^{11}\left(p_{0}\right), \\
& \operatorname{Re} \Pi_{\rho}^{a b}\left(p_{0}\right)=\left(\frac{g_{\rho}}{2 g_{\omega}}\right)^{2} \operatorname{Re} \Pi_{\omega}^{a b}\left(p_{0}\right), \quad a, b=1,2,3,
\end{aligned}
$$

whereas there is a change of sign for

$$
\begin{aligned}
& \operatorname{Re} \Pi_{\omega \rho}^{a b}\left(p_{0}\right)= \pm \frac{g_{\rho}}{2 g_{\omega}} \operatorname{Re} \Pi_{\omega}^{a b}\left(p_{0}\right), \quad a, b=1,2,3 \\
& \operatorname{Re} \Pi_{\pi \rho}\left(p_{0}\right)= \pm \frac{g_{\rho}}{2 g_{\omega}} \operatorname{Re} \Pi_{\pi \omega}\left(p_{0}\right)
\end{aligned}
$$

corresponding to the upper (lower) sign to protons (neutrons). 
The effective chemical potential $\tilde{\mu}$ is related with the thermodynamical chemical potential by means of $\tilde{\mu}_{n, p}=\mu_{n, p}-g_{\omega} W \pm g_{r} R / 2$.

[1] D. Lai, Rev. Mod. Phys. 73 (2001) 629.

[2] V. A. Miransky and I. A. Shovkovy, Phys. Rep. 576 (2015) 1.

[3] R. S. Duncan and C. Thompson, Astrophys. J. 392, L9 (1992).

[4] C. Thompson and R. S. Duncan, Mon. Not. R. Astron. Soc. 275 (1995) 255; Astrophys. J. 473 (1996) 322.

[5] M. Ruderman, Annu. Rev. Astron. Astrophys. 10, 427 (1972).

[6] C. Thompson and R. C. Duncan, Astrophys. J. 408, 194 (1993).

[7] I. Vidaña, A. Polls, and V. Durant, Phys. Rev. C 94, 054006 (2016).

[8] D. Kharzeev, Phys. Lett. B 633 (2006) 260; D. E. Kharzeev, L. D. McLerran, and H. J. Warringa, Nucl. Phys. A803, 227 (2008).

[9] Y.-J. Mo, S.-Q. Feng, and Y.-F. Shi, Phys. Rev. C 88, 024901 (2013).

[10] V. V. Skokov, A. Y. Illarionov, and V. D. Toneev, Int. J. Mod. Phys. A 24, 5925 (2009).

[11] K. Fukushima and Y. Hidaka, Phys. Rev. Lett. 117, 102301 (2016).

[12] Y. Hidaka and A. Yamamoto, Phys. Rev. D 87, 094502 (2013); E. V. Luschevskaya and O. V. Larina, Nucl. Phys. B884, 1 (2014).

[13] C. S. Machado, R. D. Matheus, S. I. Finazzo, and J. Noronha, Phys. Rev. D 89, 074027 (2014); P. Gubler, K. Hattori, S. H. Lee, M. Oka, S. Ozaki, and K. Suzuki, Phys. Rev. D 93, 054026 (2016).

[14] T. Yoshida and K. Suzuki, Phys. Rev. D 94, 074043 (2016).

[15] M. A. Andreichikov, B. O. Kerbikov, V. D. Orlovsky, and Yu. A. Simonov, Phys. Rev. D 87, 094029 (2013).

[16] H. Liu, L. Yu, and M. Huang, Phys. Rev. D 91, 014017 (2015); R. Zhang, W.-j. Fu, and Y.-x. Liu, Eur. Phys. J. C 76, 307 (2016).

[17] Sh. Fayazbakhsh, S. Sadeghian, and N. Sadooghi, Phys. Rev. D 86, 085042 (2012); S. S. Avancini, W. R. Tavares, and M. B. Pinto, Phys. Rev. D 93, 014010 (2016).

[18] J. O. Andersen, Phys. Rev. D 86, 025020 (2012).

[19] G. Colucci, E. S. Fraga, and A. Sedrakian, Phys. Lett. B 728, 19 (2014).
[20] T. Maruyama, M. Cheoun, T. Kajino, Y. Kwon, and G. J. Mathews, Phys. Rev. D 91, 123007 (2015).

[21] S. P. Adhya, M. Mandal, S. Biswas, and P. K. Roy, Phys. Rev. D 93, 074033 (2016).

[22] R. M. Aguirre, Phys. Rev. D 95, 074029 (2017).

[23] A. Mukherjee, S. Ghosh, M. Mandal, P. Roy, and S. Sarkar, Phys. Rev. D 96, 016024 (2017).

[24] M. Kawaguchi and S. Matsuzaki, Phys. Rev. D 93, 125027 (2016).

[25] S. Ghosh, A. Mukherjee, M. Mandal, S. Sarkar, and P. Roy, Phys. Rev. D 94, 094043 (2016).

[26] B. D. Serot and J. D. Walecka, Adv. Nucl. Phys. 16, 1 (1986).

[27] S. Chakrabarty, D. Bandyopadhyay, and S. Pal, Phys. Rev. Lett. 78, 2898 (1997).

[28] A. Broderick, M. Prakash, and J. M. Lattimer, Astrophys. J. 537, 351 (2000).

[29] R. Mallick, R. Gopal, S. J. Ghosh, S. Raha, and S. Roychowdhury J. Phys. G 39, 095201 (2012).

[30] J. Dong, U. Lombardo, W. Zuo, and H. Zhang, Nucl. Phys. A898, 32 (2013).

[31] J. Dong, W. Zuo, and J. Gu, Phys. Rev. D 87, 103010 (2013).

[32] R. Aguirre, E. Bauer, and I. Vidaña, Phys. Rev. C 89, 035809 (2014).

[33] A. Rabhi, M. A. Perez-Garcia, C. Providencia, and I. Vidaña, Phys. Rev. C 91, 045803 (2015).

[34] Z. Rezaei and G. H. Bordbar, Eur. Phys. J. A 52, 132 (2016).

[35] L. Tolos, M. Centelles, and A. Ramos, Astrophys. J. 834, 3 (2017).

[36] J. Alford and M. Strickland, Phys. Rev. D 88, 105017 (2013); C. Bonati, M. D'Elia, and A. Rucci, Phys. Rev. D 92, 054014 (2015).

[37] C. J. Horowitz and J. Piekarewicz, Phys. Rev. C 66, 055803 (2002).

[38] B. G. Todd-Rutel and J. Piekarewicz, Phys. Rev. Lett. 95, 122501 (2005).

[39] J. Schwinger, Phys. Rev. 82, 664 (1951).

[40] A. Chodos, K. Everding, and D. A. Owen, Phys. Rev. D 42, 2881 (1990).

[41] R. Aguirre and A. L. De Paoli, Eur. Phys. J. A 52, 343 (2016). 